\title{
Seismic wavefield polarization - Part II: Definition of a parameter system in three-dimensional (3D) space, example case review using LSBB seismic station data
}

\author{
Claire Labonne ${ }^{1,2, a}$, Olivier Sèbe ${ }^{1}$, Stéphane Gaffet ${ }^{2,3}$, François Schindelé $^{1}$, Daniel Boyer ${ }^{3}$, \\ Jean-Baptiste Decitre ${ }^{3}$, and Alain Cavaillou ${ }^{3}$ \\ ${ }^{1}$ CEA, DAM, DIF, 91297 Arpajon, France \\ ${ }^{2}$ Univ. Nice Sophia Antipolis, CNRS, IRD, Observatoire de la côte d'Azur, Géoazur UMR 7329, \\ Valbonne, France \\ ${ }^{3}$ LSBB UMS 3538, Rustrel, France
}

\begin{abstract}
A full polarization parameter system in 3D space is presented to characterize the state of polarization of a seismic wavefield and to parametrize any type of elliptical polarized seismic wave including extreme linear and circular polarizations. This parameter system does not require the a-priori knowledge of the orientation of the polarization plane and provides access to all parameters required in most polarization studies. Two groups of angular and vectorial parameters are defined, which can be easily related to one another. The knowledge of particle motion polarization opens the field of applications for 3-component seismic records contributing to improvements in phase identification. An example of a time-frequency visualization of the polarization parameters on a teleseimic earthquake recorded at a seismic station of LSBB is presented.
\end{abstract}

\section{Introduction}

A 3-component seismogram corresponds to the local trajectory of a particle motion at the surface as recorded through the passing of the seismic wavefield. The particle motion trajectory varies with the type of wave. For example, the particle motion for a P-wave is linear along the direction of propagation and for an S-wave is orthogonal to the direction of propagation. The polarization is defined as a particle motion shape and orientation. The change in polarization can be analyzed and interpreted to improve our understanding of both the source process and the Earth structures illuminated by the recorded waves. The analysis of the polarization in seismology was traditionally performed by simple visual approach using hodochrones and displays of the 3-component seismogram. Still more quantitative approaches were developed e.g. [1-5]. However, each author used his own parameters and appellations depending on the type of wave to analyse. In this paper, we bring together and standardize the different polarization parameters in order to (i) characterize the state of polarization and (ii) model the particle motion for all types of polarization. The characterization and the modelling require a system of parameters fit to describe an object

\footnotetext{
${ }^{\text {a }}$ Corresponding author: claire.labonne@cea.fr
} 
and its attributes in space. Numerous fields of study share this need. In the following, a full parameter system for seismology will be proposed based on the conclusions from [6] reviewing different parameter systems.

\section{Seismic wave polarization parameter system}

Starting the definition of the parameter system, the origin is defined at the center of the ellipse and the reference axis system is set to (North, East, Down).

To orientate an ellipse, a unique reference point needs to be chosen. Following [3], the positions of major displacement along the ellipse are ideal candidates. Still a criterion to discriminate between the two positions is needed since only one point can serve as reference. The "positive" major displacement defines the unique position of major displacement with a strictly positive Down-component i.e. the point is located under the reference plane. If the ellipse is parallel to the reference plane i.e. horizontal, this definition reaches a singularity which requires the introduction of additional criteria:

- In case of nullity of Down-component, the major displacement with strictly positive North-component is chosen.

- In case of nullity of both Down- and North-component, the major displacement with strictly positive East-component is chosen.

In case of purely circular motion, the notion of major displacement is irrelevant. This displacement has no orientation.

Deriving directly from the definition of the positive major displacement position, four parameters are defined:

- $\overrightarrow{\boldsymbol{a}}$ vector: the "positive" major displacement position vector. $|\vec{a}|$, written as $\boldsymbol{a}$, is equal to the semi-major axis length. $\vec{a}$ corresponds to the position on the ellipse at the time $t_{a}$.

- $\overrightarrow{\boldsymbol{b}}$ vector: the minor displacement vector with respect to the direction of rotation. $|\vec{b}|$, written as $\mathbf{b}$, is equal to the semi-minor axis length. $\vec{b}$ corresponds to the position on the ellipse at $t_{a}+\mathrm{T} / 4$, with $\mathrm{T}$ the rotation period.

- Plunge $\Delta$ : the angle from horizontal to the "positive" major displacement vector $(\vec{a})$.

- Trend $\Omega$ : the azimuth from North of the "positive" major displacement vector $(\vec{a})$.

Note that similar angular parameters to orientate the major displacement were also called dip or inclination and strike or azimuth by others authors as $[1,4,5]$, not to be mistaken for the parameters defined in the next paragraph to orientate the polarization plane.

To orientate the ellipse plane and take into account the direction of rotation, an "oriented" ellipse plane shall be defined. Following the astrodynamics convention, the line of intersection between the ellipse plane and the horizontal plane is called the line of nodes. The ascending node is the point where a particle following the ellipse crosses the horizontal plane downward. In case of horizontal polarization plane, the notion of node is irrelevant. Any inclined plane in 3D space can be oriented using the following two angles:

- Dip $\delta$ : the angle between the horizontal plane and the ellipse plane.

- Strike $\boldsymbol{\theta}$ : the azimuth from North of the ascending node.

A third independent angle, the rake $\gamma$, can be introduced to define directly the major direction relatively to the ellipse plane. It represents the angle between the ascending node and the 

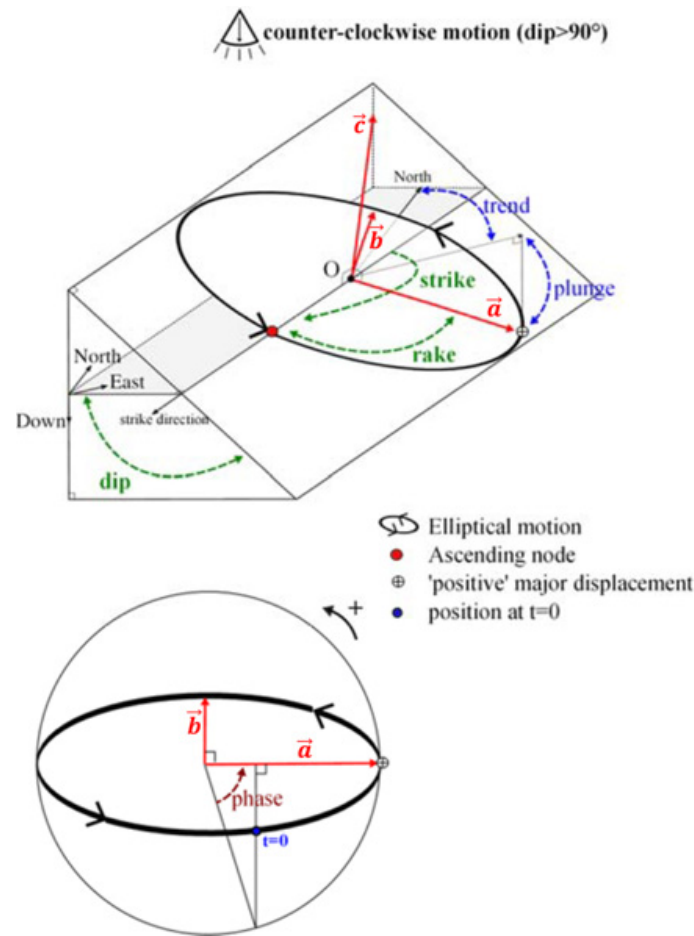
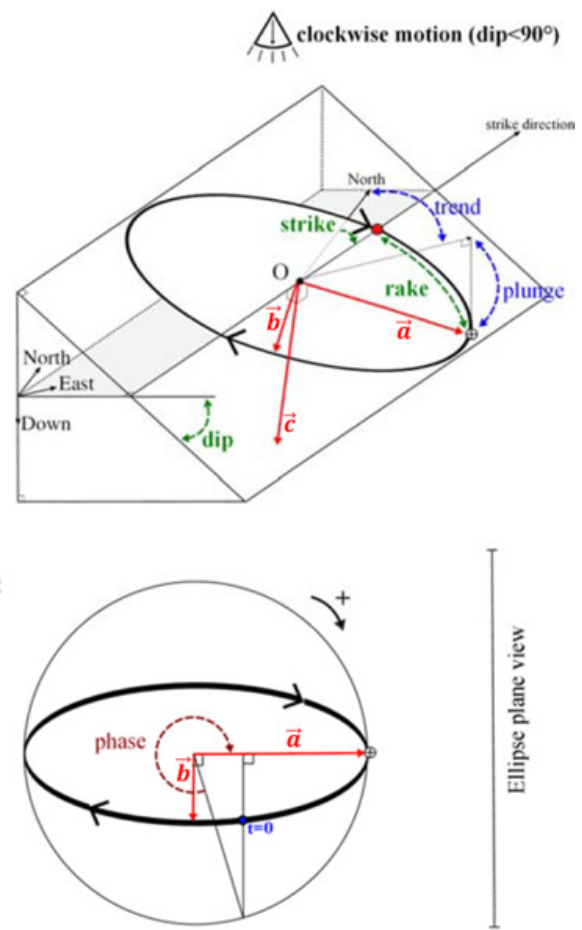

Figure 1. Seismic wave polarization parameter system.

"positive" major displacement. Note that these three angles depend on the direction of rotation. The triplets $(\delta, \theta, \gamma)$ and $(\pi-\delta, \theta+\pi, \pi-\gamma)$ correspond to the same plane but define each a different direction of rotation (see Fig. 1).

To resolve the horizontal plane singularity, a vectorial approach is preferable to the angular approach above. The vectorial approach defines the following $\overrightarrow{\boldsymbol{c}}$ vector, which is the normal vector to the ellipse plane taking into account the direction of rotation: $\vec{c}=\vec{a} \wedge \vec{b}=$ $\vec{p} \wedge \vec{q}$. It presents the strong advantage of being a null vector in case of linear motion. The orientation of the polarization is fully controlled by the orientation of $\vec{a}$ and $\vec{c}$ vectors in case of purely elliptical motion, by $\vec{a}$ vector only for linear motion and by $\vec{c}$ vector only for circular motion.

As the angular and vectorial parameters are defined on the same basis, both group of parameters can easily be related to one another by the following equations:

$$
\begin{gathered}
\text { trend }=\operatorname{atan} 2\left(a_{E}, a_{N}\right) \\
\text { plunge }=\operatorname{asin}\left(\frac{a_{D}}{|\vec{a}|}\right) \\
\text { strike }=\operatorname{atan2}\left(c_{N},-c_{E}\right) \\
\text { dip }=\operatorname{acos}\left(\frac{c_{D}}{|\vec{c}|}\right)
\end{gathered}
$$

where $\vec{a}=\left(a_{N}, a_{E}, a_{D}\right)$ and $\vec{c}=\left(c_{N}, c_{E}, c_{D}\right)$ in the (North, East and Down) system, atan2 is the four-quadrant inverse tangent. 
Table 1. List of polarization parameters and their domain of validity.

\begin{tabular}{|c|c|c|c|}
\hline \multirow[b]{2}{*}{ PARAMETER } & \multicolumn{3}{|c|}{ Particle motion } \\
\hline & LINEAR & PURELY ELLIPTICAL & CIRCULAR \\
\hline$\vec{a}$ vector & unique & unique & \multirow[t]{2}{*}{$(\vec{a}, \vec{b})$ non unique } \\
\hline$\vec{b}$ vector & $\vec{b}=\overrightarrow{0}$ & unique & \\
\hline $\overrightarrow{\mathbf{c}}$ vector & $\overrightarrow{\mathrm{c}}=\overrightarrow{0}$ & unique & unique \\
\hline Phase $\varphi$ & \multicolumn{2}{|r|}{$0 \leq \varphi<2 \pi$} & $\varphi$ depends on the choice of $\vec{a}$ \\
\hline$a$ & & $a>0$ & \\
\hline$b$ & $b=0$ & $0<b<\mathrm{a}$ & $b=a$ \\
\hline Ellipticity $\rho=\frac{b}{a}$ & $\rho=0$ & $\mathbf{0}<\boldsymbol{\rho}<\mathbf{1}$ & $\rho=1$ \\
\hline Dip $\delta$ & undefined & \multicolumn{2}{|c|}{$\mathbf{0} \leq \boldsymbol{\delta} \leq \boldsymbol{\pi}$} \\
\hline Strike $\theta$ & undefined & \multicolumn{2}{|c|}{$\mathbf{0} \leq \boldsymbol{\theta}<\mathbf{2} \boldsymbol{\pi}$ undefined if $\boldsymbol{\delta}=0$} \\
\hline Rake $\gamma$ & undefined & $0 \leq \gamma \leq \pi$ undefined if $\boldsymbol{\delta}=0$ & undefined \\
\hline Plunge $\Delta$ & & $\mathbf{0} \leq \boldsymbol{\Delta} \leq \frac{\pi}{2}$ & undefined \\
\hline Trend $\Omega$ & & $\mathbf{0} \leq \mathbf{\Omega}<\mathbf{2 \pi}$ & undefined \\
\hline
\end{tabular}

Angular and vectorial approaches can both fully describe an ellipse in 3D Space. They, however, present different advantages and drawbacks. The vectorial approach presents the major advantage of removing the singularity from the angular approach in case of a horizontal polarization plane and can easily be used to recover the angular definition (see equations above). The angular approach is closer to the usual representation of space which makes it easier to visualize.

In addition to describing the polarization orientation, the second objective of the proposed set of parameters is to characterize the shape also called ellipticity. The ellipticity parameter $(\rho)$ is defined as the ratio between $b$ and $a: \rho=\frac{b}{a}$

To reconstruct and not only characterize a polarized motion, one additional parameter needs to be defined, the phase factor $(\varphi)$. As in electromagnetism, the phase permits to locate the point on the ellipse at $\mathrm{t}=0$. The only difference is that the phase factor is an "orientated" angle relatively to the "orientated" polarization plane, meaning that the phase angle is in the same direction than the direction of rotation. Consider a circumscribed circle with a radius of $a$, that is concentric with an ellipse of semi-major axis $a$. A line perpendicular to the $\vec{a}$ vector is extended through the point at $\mathrm{t}=0$ and intersect the circle. The phase factor is the angle between the intersect point and the $\vec{a}$ vector, also called the eccentric angle. The phase factor $\varphi$ is the eccentric angle between the position at $t=0$ and the position of "positive" major displacement. This definition of the phase becomes irrelevant in case of circular motion because the notion of position of "positive" major displacement is irrelevant in such case.

All the parameters defined in this section are listed in the Table below, which summarizes the domain of validity of each parameter. The parameters that can be used to characterize the state of polarization (the shape or the orientation) are in bold.

To parametrize an elliptical motion in 3D space, the angular and vectorial approaches are equally valid and both require a set of six parameters. The six parameters proposed in case of the angular convention are the semi-major axis, the ellipticity, the dip, the strike, the rake and the phase factor, leading to the following parametrization [3]:

$$
\begin{aligned}
{\left[\begin{array}{l}
x_{N} \\
x_{E} \\
x_{D}
\end{array}\right](t)=} & {\left[\begin{array}{ccc}
\cos \theta & -\sin \theta & 0 \\
\sin \theta & \cos \theta & 0 \\
0 & 0 & 1
\end{array}\right]\left[\begin{array}{ccc}
1 & 0 & 0 \\
0 & \cos \delta & -\sin \delta \\
0 & \sin \delta & \cos \delta
\end{array}\right]\left[\begin{array}{ccc}
\cos \gamma & -\sin \gamma & 0 \\
\sin \gamma & \cos \gamma & 0 \\
0 & 0 & 1
\end{array}\right] } \\
& \times\left[\begin{array}{c}
a \cos (2 \pi f t-\varphi) \\
a \rho \sin (2 \pi f t-\varphi) \\
0
\end{array}\right]
\end{aligned}
$$



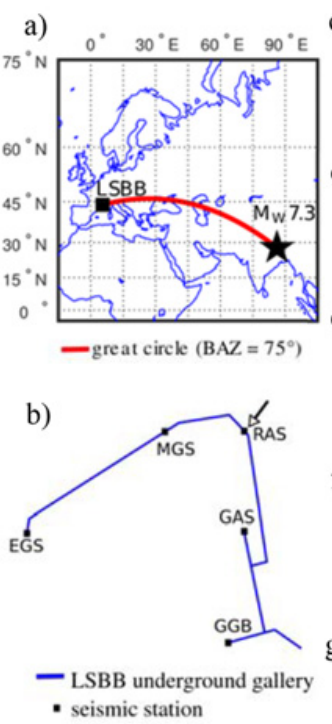

c)

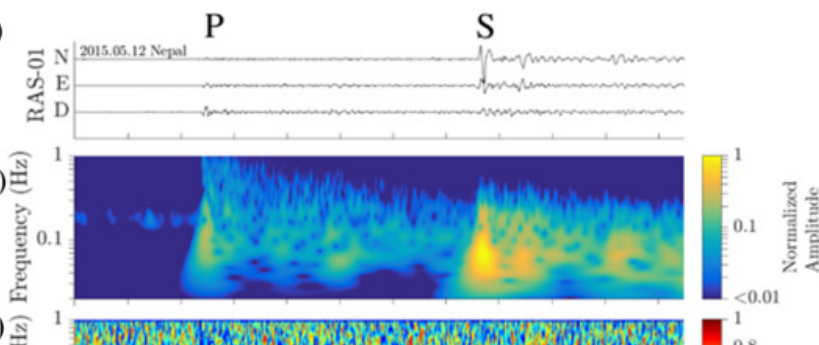

e) 졸

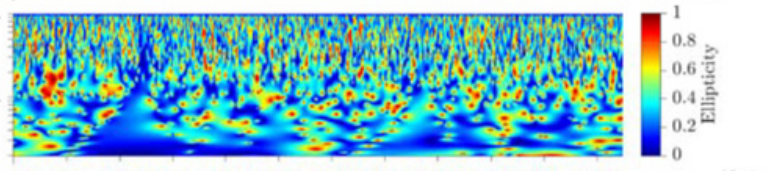

f)

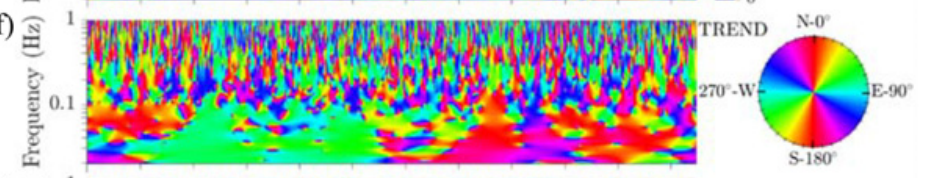

g)

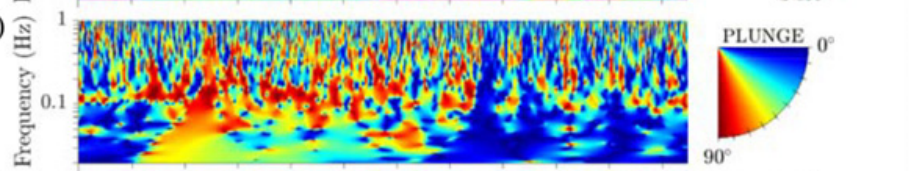

h)

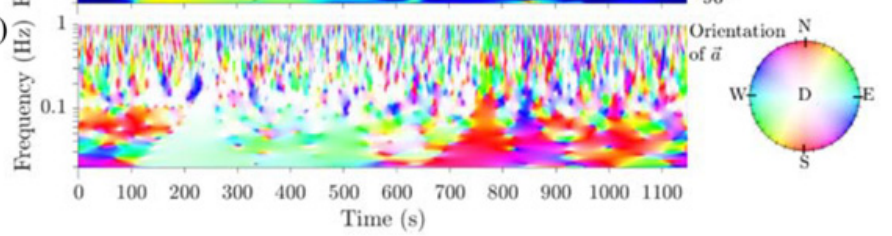

Figure 2. (a) Map showing the position of the earthquake relatively to the LSBB. (b) Map of LSBB seismic array, the arrow points the station used in this article named RAS (c) 3-component seismogram, $\mathrm{N}$ (North), E (East), D (Down) (d) Normalized amplitude spectrum obtained from an average of the 3-component Stockwell spectra (e) Ellipticity spectrum (f) Trend spectrum (g) Plunge spectrum (h) $\vec{a}$ vector orientation spectrum.

In case of the vectorial convention, the six parameters are the five independent components of the orthogonal vectors $\vec{a}$ and $\vec{c}$ and the phase factor, leading to the following parametrization:

$$
\left[\begin{array}{l}
x_{N} \\
x_{E} \\
x_{D}
\end{array}\right](t)=\vec{a} \cos (2 \pi f t-\varphi)+\underbrace{\vec{c} \wedge \vec{a}}_{\vec{b}} \sin (2 \pi f t-\varphi)
$$

This parametrization can be used to rebuild a polarized motion from information extracted from records.

\section{Visualization and comparison of polarization parameters through an earthquake record at LSBB seismic station}

To illustrate the parameter system defined above, a short study of the polarization of a seismic wavefield recorded on a Low Noise Underground Laboratory (LSBB, Laboratoire Souterrain à Bas Bruit) seismic station is presented in the following section. The example data correspond to the Mw 7.3 earthquake that occurred on May $12^{\text {th }} 2015$ in Nepal recorded by RAS station (see Fig. 2a and 2b). The epicentral distance and the back-azimuth associated to the recording station are respectively $77^{\circ}$ and $75^{\circ}$. Figure $2 \mathrm{c}$ displays a zoomed-in section of the seismogram focusing on the first arrivals of P- and S- waves. 
Following the time-frequency polarization analysis of [3], consisting in the Stockwell time-frequency decomposition (Fig. 2d) and in the approximation of each time-frequency contribution by an elliptical motion lying in a plane in the 3-D space, the polarization parameters are estimated directly from the 3-component data. Some of these parameters are represented in Fig. 2e-f-g-h, the ellipticity, the trend, the plunge, and $\vec{a}$ vector.

Assuming a 1D Earth model, the direct ray path from the epicentre to the recording station will have an azimuth of $75^{\circ}$ and an incidence of $20^{\circ}$. For the direct P-wave, the major particle displacement direction is expected to be parallel to the ray path, corresponding to respectively a trend and the plunge of $77^{\circ}$ and $70^{\circ}$. Low ellipticity values specific to linear motion (lower than 0.3 ), trend and plunge of approximatively $80^{\circ}$ and $60-90^{\circ}$ are observed at the direct $\mathrm{P}$ wave arrival time of $250 \mathrm{~s}$ roughly matching the expectations from the 1D Earth model.

For the direct $\mathrm{S}$-wave, the major particle displacement direction is expected to be orthogonal to the ray path. In Fig. 2, the direct S-wave is observed at $\sim 775 \mathrm{~s}$ on the seismogram with quasi-horizontal North-South particle motion, corresponding to an orthogonal direction to the expected ray path.

Figure $2 \mathrm{~h}$ displays the orientation of the $\vec{a}$ vector. The hue corresponds to the trend and the saturation level to the plunge. This representation of $\vec{a}$ vector orientation regroups in only one figure the information of both trend and plunge. Similarly to $\vec{a}$ vector, a representation of the orientation of $\vec{c}$ vector can also regroup the information of dip and strike.

\section{Conclusion}

Polarization is an important property that can be used to better characterize both source and Earth structure from seismic recordings. Polarization can be used to further improve the extraction, the visualization, the filtering, and the rebuilding of the polarized seismic wavefield in $3 \mathrm{D}$ space. An exhaustive and simple parameter system is an absolute prerequisite to the description of the polarization. Two equivalent groups of parameters have been defined, angular and vectorial parameters. The angular parameters can be deduced from the vectorial parameters. To fully characterize the polarization, both shape and an orientation should be described in space. The shape is fully controlled by the ellipticity parameter. Using the vectorial parameter system, the orientation of the polarization is fully controlled by the orientation of the $\vec{a}$ and $\vec{c}$ vectors in case of purely elliptical motion, by the $\vec{a}$ vector only for linear motion and by the $\vec{c}$ vector only for circular motion. The vectorial parameters have the strong advantage to reduce the number of parameter to visualize.

This work was performed through the funding of the "Région Provence Alples Côte d'Azur" (PACA) and the Commissariat à l'Energie Atomique et aux Energies Alternatives (CEA). Authors thank the LSBB team and the Technologie de la Mesure dans la Géosphère (CEA/TMG) staff for their technical help.

\section{References}

[1] Vidale, J.E. (1986), Complex polarization analysis of particle motion. Bulletin of the Seismological society of America, 76(5), 1393-1405.

[2] Diallo, M.S., Kulesh, M., Holschneider, M., Scherbaum, F., \& Adler, F. (2006), Characterization of polarization attributes of seismic waves using continuous wavelet transforms. Geophysics, 71(3), V67-V77.

[3] Pinnegar, C.R. (2006), Polarization analysis and polarization filtering of threecomponent signals with the time-frequency $\mathrm{S}$ transform. Geophysical Journal International, 165(2), 596-606. 
[4] Jurkevics, A. (1988), Polarization analysis of three-component array data. Bulletin of the Seismological Society of America, 78(5), 1725-1743.

[5] Kulesh, M., Diallo, M.S., Holschneider, M., Kurennaya, K., Krüger, F., Ohrnberger, M., \& Scherbaum, F. (2007), Polarization analysis in the wavelet domain based on the adaptive covariance method. Geophysical Journal International, 170(2), 667-678.

[6] Labonne, C., Sèbe, O., Gaffet S. (2016), Seismic wavefield polarization - Part I: Describing an elliptical polarized motion, a review of motivations and methods. 